\title{
EDITORIAL
}

\section{„Doing spatial research“}

\author{
Gregor Prinzensing
}

Online publiziert: 16. Mai 2015

(C) Springer-Verlag Berlin Heidelberg 2015

\section{Liebe Leserinnen und Leser,}

die vorliegende Ausgabe der „Raumforschung und Raumordnung" zeigt mit ihren facettenreichen Beiträgen einmal mehr die im besten Sinne erstaunliche Diversität raumwissenschaftlicher Forschung.

Das Heft wird eröffnet mit einem Beitrag aus der Rubrik „Zur Diskussion“. Hier veröffentlichen wir bekanntlich in unregelmäßiger Folge fachliche „Zwischenrufe“, in denen sich eine vielleicht manchmal zugespitzte Position artikuliert und so - mehr noch als in anderen Beiträgen - auf Einrede und eine insgesamt weiterführende Diskussion rechnet. Timm Sebastian Wiegand und Hans Heinrich Blotevogel widmen sich hierzu dem Verhältnis von Wissenschaft und Praxis und besonders den Prozessen der Wissensgenerierung und des Wissenstransfers in transdisziplinären Kontexten. Am Beispiel der Arbeit der Akademie für Raumforschung und Landesplanung (ARL) untersuchen sie, wie die konkreten praktischen Wirkungen, die von wissenschaftlichen Projekten und ihren Produkten und Ergebnissen ausgehen, ermittelt werden können. Insbesondere die Frage, wie die Würdigung dieser Wirkungen dann gegebenenfalls im Rahmen etwa der wissenschaftlichen Evaluierung einer Einrichtung ausfallen kann, findet besondere Beachtung durch die Autoren. Bislang haben sich solche Evaluierungen ja in erster Linie dem Zweck der Beförderung wissenschaftlicher Exzellenz verschrieben. Wer für die Bedürfnisse der

G. Prinzensing $(\bowtie)$

Akademie für Raumforschung und Landesplanung (ARL) -

Leibniz-Forum für Raumwissenschaften,

Hohenzollernstraße 11,

30161 Hannover, Deutschland

E-Mail: prinzensing@arl-net.de raumwissenschaftlichen Praxis forscht und arbeitet, weiß hingegen, dass mit den Maßstäben der Exzellenz hier in der Regel nicht viel zu bewegen ist. Der Beitrag versteht sich als Denkanstoß, in welcher Richtung die Leistungsmessung von praxisorientierten wissenschaftlichen Institutionen weiter zu entwickeln wäre.

Der anschließende Beitrag von Frank Osterhage, ClausChristian Wiegandt und Stefan Haunstein unternimmt am Beispiel dreier Stadtregionen (Köln/Bonn, Östliches Ruhrgebiet und Leipzig/Halle) eine vergleichende Erforschung und Visualisierung polyzentrischer Siedlungsstrukturen. Dazu werden die jeweiligen funktionsräumlichen Differenzierungen der Stadträume, dann besonders die Siedlungsstrukturen im Bereich des Wohnens und schließlich die Pendlerverflechtungen zwischen den Zentren und ihrem jeweiligen Umland (auch historisch) untersucht. Ein für die Raumwissenschaften zentrales Medium - die Karte wird dabei gleichwertig neben die textliche Darstellung und sprachlich-analytische Aufgliederung der betrachteten Räume gestellt. Die Karten sind hierbei nicht bloße Medien der Veranschaulichung dessen, was sich ohnehin schon aus dem Text ergibt, sondern selbst ein zentrales Ergebnis des Beitrags mit einem eigenständigen Beweiswert. Raumstruktur zeigt sich insgesamt als ein Resultat längerfristiger Entwicklungen, wobei den traditionellen Siedlungskernen allerdings weiterhin hohe Bedeutung zukommt.

Im Beitrag von Manfred Kühn und Ulrike Milstrey wird ebenfalls vergleichend zu Stadtregionen gearbeitet. Zwei Fallstudien in schrumpfenden und peripheren Regionen - Beispiele sind hier das Städtedreieck Altmark (SachsenAnhalt) und die Standortinitiative Südwestpfalz (Rheinland-Pfalz) - beleuchten Versuche von interkommunaler Kooperation unter dem Einfluss von Städtekonkurrenz im Rahmen zentralörtlicher Hierarchien. Ein elaboriertes Konzept von regional governance bildet dabei die Folie zur 
Gegenüberstellung und Diskussion der Ergebnisse. Für kommunale Akteure insbesondere in peripheren Regionen entsteht unter Schrumpfungsbedingungen offenbar eine paradoxe Gleichzeitigkeit von Kooperations- und Konkurrenzdruck, die je und je andere Verschränkungen beider Imperative (quasi Spielarten von „co-opetition“) herbeiführt. Dabei kann eine funktionale Arbeitsteilung zwischen Kommunen die Rahmenbedingungen für interkommunale Kooperationen durchaus begünstigen, auch wenn die Frage nach dem geeigneten räumlichen Maßstab dafür letztlich offen bleibt.

Der Beitrag von Robert Nadler, Knut Petzold und Robert Schönduwe widmet sich der methodisch interessanten Fragestellung, inwieweit Online-Befragungen inzwischen ein gängiges und gewöhnlich genutztes Werkzeug aus dem „Besteckkasten“ der empirischen Sozial- und insbesondere auch der raumwissenschaftlichen Forschung sind. Mit Hilfe verschiedener Beispiele diskutieren sie Fragen der spezifischen Methodologie und zeigen Vor- und Nachteile sowie mögliche Einsatzfelder des Erhebungsinstrumentes auf. Insbesondere bei der Erforschung hochmobiler Bevölkerungsgruppen können Online-Befragungen vermutlich ein wertvolles Hilfsmittel sein. Neben einer Darstellung des geeigneten Vorgehens bei der Fragebogengestaltung, beim Sampling und bei der technischen Umsetzung stellen die Autoren auch ausgewählte eigene Ergebnisse vor und ergänzen diese um einen Katalog allgemeiner Fragen, deren Beantwortung die Entscheidung über den Einsatz einer Online-Befragung als Methode erleichtert. Nach ihrer Ansicht bieten Online-Befragungen in den Raumwissenschaften ein noch zu wenig genutztes Potenzial zur Erforschung von Mobilitäts- und Multilokalitätsphänomenen.

Der „Bericht aus Forschung und Praxis“ von Sascha Anders schließlich stellt wesentliche Ergebnisse aus dem Projekt „Qualifizierte Nahversorgung im Lebensmitteleinzelhandel" dar. Hier wurden aus planungs- bzw. genehmigungsrechtlicher Sicht unter anderem Einzugsbereiche und verkehrliche Wirkungen der unterschiedlichen Betriebsformen im deutschen Lebensmitteleinzelhandel analysiert. Die Studie ging - empirisch auf eine bundesweit durchgeführte telefonische Haushaltsbefragung gestützt - der Frage nach, ob generalisierbare Aussagen möglich sind zu den zu erwartenden Wirkungen, die von neuen Lebensmittel- und Supermärkten ausgehen - etwa abhängig von ihrer geplanten Verkaufsfläche. Neben der reinen Größe des Marktes beeinflussen aber anscheinend vor allem die Lage (städtebaulich integriert vs. städtebaulich nicht integriert), die umgebende Siedlungsstruktur (städtisch vs. ländlich geprägt), aber auch die Anbieter selbst mit ihren unterschiedlichen Expansionsund Marketingkonzepten die verkehrlichen Auswirkungen und die Reichweite der Kaufkraftabschöpfung entscheidend.

Wir wünschen unseren Leserinnen und Lesern mit diesen Artikeln wie immer eine kurzweilige und anregende Lektüre. 